\title{
Líquidos Cerebroespinal, Cefalorraquídeo o Encéfaloespinal. Una Perspectiva Holística para Terminologia Anatomica
}

\author{
Cerebrospinal, Cephalic-rachid or Encephalospinal Fluids. \\ A Holistic Perspective for Terminologia Anatomica
}

\author{
Jorge Eduardo Duque Parra ${ }^{1,2,3}$ \& Bélgica Vásquez ${ }^{4}$
}

DUQUE-PARRA, J. E. \& VÁSQUEZ, B. Líquidos cerebroespinal, cefalorraquídeo o encéfaloespinal. Una perspectiva holística para Terminologia Anatomica. Int. J. Morphol., 38(5):1421-1425, 2020.

RESUMEN: En el campo morfológico internacional se utiliza el término líquido cerebroespinal, identificado en latín como Liquor cerebrospinalis. En el humano, este líquido circula por varias cavidades del sistema nervioso central, de las cuales, se reconocen cinco ventrículos. Cuatro son componentes encefálicos y uno se encuentra en la parte terminal de la médula espinal. El objetivo del presente trabajo fue determinar cuál es el nombre más apropiado para este líquido, valorando el uso corriente que se da en libros de texto usados para la enseñanza de la neuroanatomía y en artículos científicos. Para ello, se efectuó una búsqueda bibliografía, no sistemática y aleatoria, de libros y artículos científicos en los cuales se hace mención sobre el fluido del sistema ventricular encefálico y el espacio subaracnoideo del ser humano. El $100 \%$ de los libros y artículos científicos publicados en inglés, utilizaron el término líquido cerebroespinal para denominar este fluido. El $90 \%$ de los libros y artículos científicos publicados en español utilizaron el término líquido cefalorraquídeo y el $10 \%$ de ellos, líquido cerebroespinal. Los resultados demuestran que en América Latina aún se utiliza más de un nombre para una misma estructura. Tanto libros como artículos científicos publicados en español no han seguido las recomendaciones de la Federación Internacional de Asociaciones de Anatomistas (IFAA), ya que la mayoría de ellos utilizó el término líquido cefalorraquídeo. Por otra parte, creemos que el término más apropiado para referirse al líquido extracelular que circula por el sistema ventricular nervioso y espacio subaracnoideo, es el de líquido encéfaloespinal. Este término se propone para suplir el vigente y tradicional líquido cerebroespinal en inglés y líquido encefalorraquídeo en español.

PALABRAS CLAVE: Líquido cerebroespinal; Líquido cefalorraquídeo; Líquido encéfaloespinal; Terminologia Anatomica; Terminologia Neuronatomica.

\section{INTRODUCCIÓN}

En el campo morfológico internacional se usa el término líquido cerebroespinal, identificado como Liquor cerebrospinalis en latín y "cerebrospinal fluid" en inglés, con el número 266 en el capítulo I sobre sistema nervioso central de Terminologia Neuroanatomica (FIPAT, 2017b).

Este líquido circula por varias cavidades del sistema nervioso central, de las cuales, se reconocen cinco ventrículos. Cuatro son componentes encefálicos: dos en el cerebro, uno en el diencéfalo y otro en el tronco encefálico (Duque-Parra et al., 2017; Scelsi et al., 2020). El quinto ventrículo se encuentra en la parte terminal de la médula espinal, a nivel del cono medular de los primates, incluidos los seres humanos (Duque Parra et al., 2020).
Los primeros cuatro ventrículos, son los únicos que poseen plexos coroideos, componentes neuro conectivos vasculares, cuyo epitelio simple cuboideo (Del Bigio, 2010) presenta microvellosidades finas e irregulares que aumentan la superficie de la célula para su actividad secretora y producción de líquido cerebroespinal (Tamega et al., 2000; Melody et al., 2015). En el ser humano, este líquido se mueve dentro del sistema nervioso central, desde la región superior a la inferior, pasando de los ventrículos laterales, por los forámenes interventriculares hacia el tercer ventrículo (Scelsi et al.) y, de este, por el acueducto mesencefálico hacia el cuarto ventrículo (Scelsi et al.); luego, sale del cuarto ventrículo al espacio subaracnoideo, por tres forámenes, dos laterales y uno a nivel de la línea media (Lee et al., 2012).

\footnotetext{
${ }^{1}$ Departamento de Ciencias Básicas, Programa de Medicina, Universidad de Caldas, Colombia.

${ }^{2}$ Departamento de Ciencias Básicas, Programa de Medicina, Universidad de Manizales, Colombia.

${ }^{3}$ Grupo Neurociencia de Caldas, Colombia.

${ }^{4}$ Universidad de Tarapacá, Chile.
} 
El objetivo del presente trabajo fue determinar cuál es el nombre más apropiado para este líquido, de distribución encefálica y espinal, valorando el uso corriente que se da en libros de texto usados para la enseñanza de la neuroanatomía y en artículos científicos.

\section{MATERIAL Y MÉTODO}

Se efectuó una búsqueda bibliografía, no sistemática y aleatoria, de libros y artículos científicos en los cuales se hace mención sobre el fluido del sistema ventricular encefálico y el espacio subaracnoideo del ser humano, con el fin de conocer cuál es el término usado para denominar este fluido, verificar si se sigue o no la Terminologia Neuroanatomica (FIPAT, 2017b) y Terminologia Anatomica (FIPAT, 2019) y, posteriormente, proponer un término adecuado para denominar este fluido.

Para ello, se revisaron 10 libros, utilizados en la enseñanza de la neuroanatomía en inglés y 10 en español. Además, revisamos 10 artículos científicos en inglés presentes en la base de datos de PubMed, correspondientes al año 2020 y 10 artículos científicos en español de 1980 a 2019.

Los libros y artículos científicos seleccionados fueron registrados en tablas, ordenando las referencias alfabéticamente.

\section{RESULTADOS}

El $100 \%$ de los libros en inglés, usados para la enseñanza de la neuroanatomía, utilizó el término líquido cerebroespinal para denominar el fluido del sistema ventricular encefálico y espinal (Tabla I). De los 10 libros en español, 9 utilizaban el término líquido cefalorraquídeo y uno líquido cerebroespinal (Tabla II). El $100 \%$ de los artículos científicos publicados en inglés usaba el término líquido cerebroespinal (Tabla III) y, de los artículos publicados en español, 9 utilizaban el término líquido cefalorraquídeo y uno líquido cerebroespinal (Tabla IV).

\section{DISCUSIÓN}

La neuroanatomía es una importante área de la morfología que utiliza la Terminologia Anatomica y la Terminologia Neuroanatomica, las cuales propenden por una comunicación más clara y precisa entre los profesionales de la salud, aunque en América Latina sigue siendo un grave problema de comunicación científica entre los profesionales y estudiantes del área de la salud (Duque et al., 2016) y de las ciencias afines, pues entre otros aspectos, se utilizan diversos nombres para una misma estructura (Duque et al., 2018), como es el caso de líquido cerebroespinal y líquido cefalorraquídeo, objeto de este trabajo.

Tabla I. Libros en inglés y término usado para el fluido del sistema ventricular encefálico y espacio subaracnoideo.

\begin{tabular}{|c|c|}
\hline LIBRO - TEXTO & TÉRMINO \\
\hline $\begin{array}{l}\text { B huiyan, P. S.; Rajgopal, L.; Shyamkishore, K. \& Inderbir Singhs. Text book of neuroanatomy. Fundamental al clinical. } 9 \text { ed. } \\
\text { New Dheli, Jaypee brothers medical publishers, } 2014 .\end{array}$ & Cerebrospinal fluid \\
\hline Blumenfeld, H. Neuroanatomy through clinical cases. Massachusetts, Sinauer Associates Sunderlands, 2002. & Cerebrospinal fluid \\
\hline Douglas, J. G. \& Jennifer, K. Sidman's Neuroanatomy: a programmed learning tool. Brueckner. $2^{\text {nd }}$ ed. Lippincott Williams \& Wilkins, 2008. & Cerebrospinal fluid \\
\hline Goldberg, S. Clinical neuroanatomy made ridiculously simple. $3^{\mathrm{a}}$ ed. Miami. Medmaster, 2003. & Cerebrospinal fluid \\
\hline Haines, D. E. Neuroanatomy. An Atlas of structures, sections, and systems. 8a . ed. Philadelphia, Lippincott Williams \& Wilkins, 2012. & Cerebrospinal fluid \\
\hline Martin, J. H. Neuroanatomy text and atlas. 4 ed. New York, McGraw-Hill, 2012. & Cere brospinal fluid \\
\hline Mancall, E. L. \& Brock, D. G. Clinical Neuroanatomy. The Anatomic basis for clini cal Neuroscience. Philadelphia, Elsevier, 2011. & Cerebrospinal fluid \\
\hline Mtui, E.; Gruener, G. \& Dockery, P. FitzGerald's. Clinical neuroanatomy and neuroscience. $7^{\text {th }}$ ed. Philadelphia, Elsevier, 2016. & Cerebrospinal fluid \\
\hline Snell, R. S. Clinical neuroanatomy. $7^{\text {th. }}$ ed. Philadelp hia, Lippincott Williams \& Wilkins, 2010. & Cerebrospinal fluid \\
\hline Standring, S. Gray's Anatomy. The Anatomical basis of clinical practique. $41^{\text {th }}$ ed. Philadelphia. Elsevier, 2016. & Cerebrospinal fluid \\
\hline
\end{tabular}

Tabla II. Libros en español y término usado para el fluido del sistema ventricular encefálico y espacio subaracnoideo.

\begin{tabular}{|c|c|}
\hline LIBRO - TEXTO & TÉRMINO \\
\hline Afifi, A. K. \& Bergman, R. A. Neuroanatomía funcional texto y atlas. 2a ed. México, Mc Graw Hill. Interamericana, 2006. & Líquido cerebroespinal \\
\hline Clark, D. L.; Boutros, N. N. \& Mendez, M. F. El cerebro y la conducta neuroanatómica para psicólogos. $3^{\mathrm{a}}$ ed. México, El manual moderno, 2012. & Líquido cefalorraquídeo \\
\hline Crossman, A. R. \& Neary, D. Neuroanatomía. Texto y atlas en color. $5^{\mathrm{a}}$ ed. Barcelona, Elsevier, 2015. & Líquido cefalorraquídeo \\
\hline García-Porrero, J. A. \& Hurlé, J. M. Neuroanatomía humana. Madrid, Panamericana, 2015. & Líquido cefalorraquídeo \\
\hline Kiernan, J. A. \& Rajakumar, N. Barr. El sistema newioso humano. Una perspectiva anatómica. 10ª ed. Barcelona, Wolters Kluwer, 2014. & Líquido cefalorraquídeo \\
\hline Mtui, E.; Gruener, G. \& Dockery P. Neuroanatomía clínica y Neurociencia. $7^{\mathrm{a}}$ ed. Barcelona, Elsevier, 2017. & Líquido cefalorraquídeo \\
\hline Pró, E. A. Anatomía Clínica. 2 ed. Buenos Aires, Panamericana, 2014. & Líquido cefalorraquídeo \\
\hline Puelles López, L.; Martínez Pérez, S. \& Martínez de la Torre, M. Neuroanatomía. Madrid, Panamericana, 2008. & Líquido cefalorraquídeo \\
\hline Rubin, M. \& Safdieh, J. E. Netter neuroanatomía esencial. Barcelona, Elsevier Masson, 2008. & Líquido cefalorraquídeo \\
\hline Snell, R. S. Neuroanatomía clínica. $7^{\mathrm{a}}$ ed. Barcelona, Wolters Kluwer, 2014. & Líquido cefalorraquídeo \\
\hline
\end{tabular}


Tabla III. Artículos en inglés y término usado para el fluido del sistema ventricular encefálico y espacio subaracnoideo.

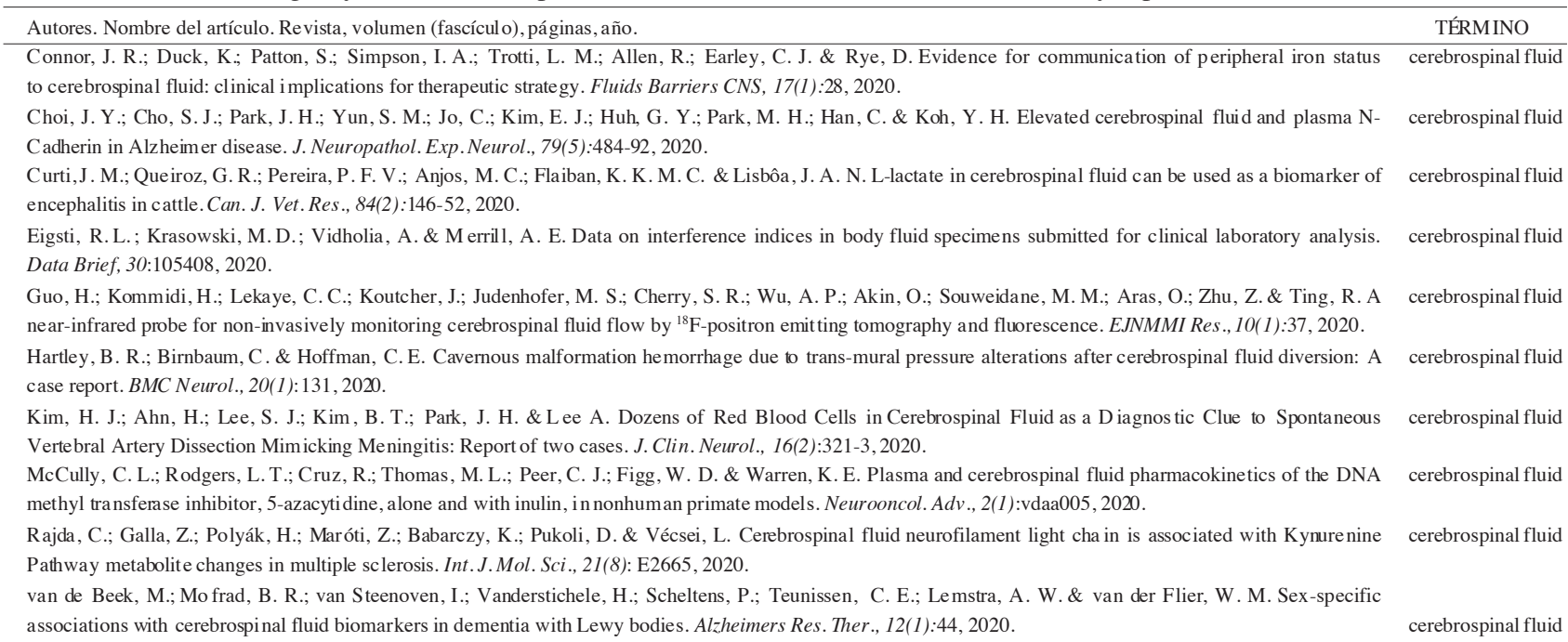

Tabla IV. Artículos en español y término usado para el fluido del sistema ventricular encefálico y espacio subaracnoideo.

Autores. Nombre del artículo. Revista, volumen (fascícul o), páginas, año.

Cabrera-Maqueda, J. M.; Fuentes-Rumí, L.; Valero-López, G.; Baidez- Guerrero, A. E.; García-Molina, E.; Díaz-Pérez, J. \& Gar cía-Vázquez, E. Líquido cefalorraquídeo Difusión de los antibióticos en el sistema nervioso central. Rev. Esp. Quimioter., 31(1): 01-12, 2018.

Duarte, M. M. S.; Camacho-Moreno, G. \& Mancilla, G. N. Valores del análisis citoquímico de líquido cefalorraquídeo en pacientes con derivación Líquido cefalorraquíd eo ventrículo-peritoneal. Rev. Chilena Infectol., 36(3):378-83, 2019

Guzmán, M.; Ayala, M.; Toro-Gonzalez, G.; Morales, L. \& Díaz, H. Estudio del líquido cefalorraquídeo en el diagnóstico de 1 a panencefalitis Líquido cefalorraquídeo esclerosante sub-aguda (peesa). Acta Med.Col., 5(3):431-7, 1980.

Martínez Brú, C.; Llompart Alabern, I. Recomendaciones para el estudio de las proteínas del líquido cefal orraquídeo. Química Analítica, 21(2):83-90, Líquido cefalorraquíd eo 2002.

Moraes, M.; Camacho, S.; Margni, C. \& Borbonet, D. Utilidad del examen de líquido cefalorraquídeo y de h radiografía de huesos largos en neonatos Líquido cefalorraquíd eo asintomáticos con riesgo de sífilis connatal. Arch. Pediatr. Urug., 84(3):187-92, 2013.

Munive, B. L. Punción lumbar. Condiciones e in dicaciones en pediatría Acta Pediátr. Mex., 35:423-7, 2014.

Líquido cefalorraquíd eo

Navarro Suay, R.; García Aroca, M. A.; López Soberón, E. \& Pelet Pascual, E. Líquido cefalorraquídeo xantocrómico, ¿cómo puede ser posible? Sanid. Líquido cefalorraquíd eo Mil., 73(4): 224-5, 2017.

Pérez-Neri, I. \& Aguirre-Espinosa, A. C. Dinámica del líquido cefalorraquídeo y barrera hematoencefálica. Arch. Neurocien. (Mex)., 20 (1):60-4, 2015. Serrano, V. S. Manifestaciones neurológicas de la infección por VIH en pediatría.Rev. Chil. Pediatr., 64(4):259-63, 1993.

Tola Arribas, M. A. Historia del líquido cefalorraquídeo: desde la Antigüedad hasta la Edad Contemporánea. Neurosci. Hist.,5(3):105-13, 2017.

Lo anterior, se debe a diversos factores, entre los que se destaca, que los textos utilizados en la enseñanza neuroanatómica no han seguido las recomendaciones de la Federación Internacional de Asociaciones de Anatomistas (IFAA), y para el presente estudio, involucra que la mayoría de los textos revisados en español utilizó el término líquido cefalorraquídeo. Esto se podría deber, a que muchos morfólogos no han aceptado el término en latín y desean seguir usando un término consagrado en español (Duque et al., 2018) o no han planteado otras opciones racionales al respecto.

Si se utiliza una terminología especializada que permite la correcta descripción de todos los componentes que conforman el sistema nervioso, dichas palabras allí empleadas, permitirían dar un fundamento para perfeccionar el diálogo con la tentativa de encontrar la equivalencia que, para el caso, usa varios términos sobre el fluido que circula por el sistema nervioso central endo ventricularmente y por el espacio subaracnoideo. Aunque el uso del término en cues- tión es apropiado en inglés, no corresponde con el original en latín de Liquor cerebrospinalis (FIPAT, 2017 y 2019).

Además, hay varias dudas: en inglés se utiliza el término líquido cerebroespinal, pero creemos que no necesariamente es el más adecuado, pues al usar este término se da referencia a que el líquido está en el cerebro, sin incluir el diencéfalo, el cerebelo y el tronco encefálico, particularizando en el componente más voluminoso del sistema nervioso central: el cerebro. Aunque en inglés, encéfalo se homologa por cerebro "brain" y se define como la parte del sistema nervioso central contenido dentro del cráneo, que comprende el prosencéfalo, mesencéfalo y rombencéfalo y sus subdivisiones (Dorland's, 2012), indicadas en textos de neurodesarrollo intrauterino, como si fueran estructuras diferentes, es decir, no parece existir comprensión ontogenética. Junto con lo anterior, en Terminología Anatomica (término 5388) y Terminologia Neuroanatomica (término 266) aparece en ambas terminología el término en- 
céfalo, en latín Encephalon, sin embargo, es traducido como "brain" -cerebro-, que los hablantes en inglés han asimilado. Los otros componentes como Truncus encephali (tronco encefálico en español) pasa al inglés como "Brain stem", que incluye el Myelencephalon (mielencéfalo), el Pons (puente) y el Mesencephalon (mesencéfalo). Además, se señala el Cerebellum (cerebelo), el Prosencephalon (prosencéfalo), traducido al inglés como "forebrain" -cerebro anterior-; el Diencephalon (diencéfalo) y el Telencephalon (telencéfalo), cuya segunda columna indica un sinónimo en latín Cerebrum (cerebro en español) (FIPAT, 2017b); sin embargo, en Terminologia Anatomica (FIPAT, 2019) este término no tiene sinónimo en latín.

Por otra parte, en inglés y español, es usado el término electroencefalograma "electroencephalogram" (Aiyer et al., 2016; Fahy \& Chau, 2018) en referencia a registrar los potenciales masivos de la actividad neuronal del cerebro. Sin embargo, considerando el análisis anterior y en sincronía con el mismo, ¿por qué no usan un término como electrocerebrograma "electrobraingram"?

En algunos artículos científicos también se usan términos como desarrollo de la corteza cerebral "cerebral cortical", cerebro humano "human brain" y microcefalia "microcephaly" (Lehtinen \& Walsh, 2011). Los términos: prosencéfalo, telencéfalo, diencéfalo, mielencéfalo y romboencéfalo se utilizan en el estudio del desarrollo intrauterino del embrión y feto que se encuentran señalados en Terminologia Embryologica (FIPAT, 2017a), ¿por qué luego se usan de manera diferente si se está reconociendo implícitamente el término céfalo para denotar cabeza? y, además, en casos de alteraciones del neurodesarrollo, no se usa el término cerebro, sino encéfalo, lo que es claro al denominar anencefalia para la afección que forma parte del espectro del defecto del tubo neural, en el que el cerebro y los huesos craneales están muy malformados, en donde las estructuras cerebrales y cerebolosas están reducidas o con malformación, sin embargo, el cerebro posterior está presente (Kumar et al., 2015; Bayat, 2016; Ekmekci \& Gencdal 2019).

En base a los análisis realizados, la palabra cerebro como parte del término líquido cerebroespinal, no considera otras estructuras por donde fluye y se encuentra este fluido. En español, si se utiliza el término cefalorraquídeo, se refiere a que está en la cabeza y no particulariza que está en su interior, ejemplo: la vena facial está en la cabeza, pero no en su interior, por lo que es una vena pericefálica y no endocefálica. Además, raquídeo hace referencia a la columna vertebral raquis- y no particulariza en su interior, es decir, en el conducto central, en el quinto ventrículo y en el espacio subaracnoideo con sus diversas cisternas durales-aracnoideas, aunque una pequeña parte sale por las raíces de los nervios espinales. Por ello, el término que proponemos para este líquido es encéfaloespinal, para referir que se ubica en el encéfalo, interior de la cabeza, tanto por el sistema ventricular como por su espacio subaracnoideo, y espinal, para referir que circula por dentro de la médula espinal y en el espacio subaracnoideo de esta. Este término parece más holístico, general y particular a la vez, para designar este fluido del sistema nervioso central, por lo que sugerimos implementarla en Terminologia Anatomica y Terminologia Neuroanatomica.

\section{CONCLUSIÓN}

El término más apropiado para referirse al líquido extracelular que circula por el sistema ventricular nervioso y espacio subaracnoideo es el de líquido encéfaloespinal. Se propone para suplir el vigente y tradicional líquido cerebroespinal en inglés y líquido encefalorraquídeo en español.

DUQUE-PARRA, J. E. \& VÁSQUEZ, B. Cerebrospinal, Cephalic-rachid or encephalospinal fluids. A holistic perspective for Terminologia Anatomica. Int. J. Morphol., 38(5):1421-1425, 2020 .

SUMMARY: The term cerebrospinal fluid is generally used in morphology. In Latin it is known as liquor cerebrospinalis. In the human, this fluid circulates through various cavities of the central nervous system, of which, five ventricles are recognized. Four are brain components and one is in the terminal part of the spinal cord. The objective of the present study was to determine the most appropriate term for this liquid, while evaluating the current use of terminology in scientific publications and in academic textbooks used to tech neuroanatomy. A non-systematic and randomized bibliographic search of books and scientific articles was carried out, making mention of the encephalic ventricular system fluid and the subarachnoid space of the humans. A total, $(100 \%)$ of identified scientific books and articles published in English, used the term cerebrospinal fluid to refer to this fluid; $90 \%$ of the scientific books and articles published in Spanish used the term cephalic-rachid fluid; cerebrospinal fluid was used in $10 \%$ of these. The results show that more than one term continues to be used in Latin America to refer to this structure. International Federation of Anatomical Associations (IFAA) recommendations have not been followed, since most books and scientific research publications use the term cerebrospinal fluid to describe the structure. Moreover, we believe that the cephalic-spinal fluid is the most appropriate term in this case, given that circulation occurs throughout the ventricular nervous system and the subarachnoid space. This term is proposed to replace the current and traditional cerebrospinal fluid in English and cephalic-rachid fluid in Spanish.

KEY WORDS: Cerebrospinal fluid; Cerebrospinal fluid; Brain-spinal fluid; Terminologia Anatomica; Terminologia Neuroanatomica. 


\section{REFERENCIAS BIBLIOGRÁFICAS}

Aiyer, R.; Novakovic, V. \& Barkin, R. L. A systematic review on the impact of psychotropic drugson electroencephalogram waveforms in psychiatry. Postgrad. Med., 128(7):656-64, 2016.

Bayat, P. D. Abnormities in boy anencephalic newborn in Iran. J. Morphol. Sci., 33(4):197-9, 2016.

Del Bigio, M. R. Ependymal cells: biology and pathology. Acta. Neuropathol., 119(1):55-73, 2010.

Dornald. Dorland's illustrated medical dictionary. $32^{\text {nd }}$ ed. Elsevier, Saunders, 2012.

Duque Parra, J. E.; Aguirre, M. A. \& Vélez García J. F. The terminal ventricle in Saguinus leucopus. J. Anat., 236.1:P2-V3, 2020.

Duque, P. J. E.; Barco Ríos, J. \& Aldana Rueda, J. E. La terminología anatómica en Colombia y el uso de epónimos en la enseñanza en medicina. Rev. Biosalud, 15(1):82-6, 2016.

Duque-Parra, J. E.; Barco-Ríos, J. \& García-Aguirre, J. F. A historical approach to the ventricular system of the brain. Rev. Fac. Med., 65(3): 473-7, 2017.

Duque, P. J. E.; Vásquez, B. \& del Sol, M. Problemática Educativa Terminológica Anatómica en América latina: Terminologia Anatomica es Internacional, no Nacional ni Regional. Int. J. Morphol., 36(4):142330, 2018.

Ekmekci, E. \& Gencdal, S. What's happening when the pregnancies are not terminated in case of anencephalic fetuses? J. Clin. Med. Res., 11(5):332-6, 2019.

Fahy, B. G. \& Chau, D. F. The Technology of Processed electro encephalogram monitoring devices for assessment of depth of anesthesia. Anesth. Analg., 126(1):111-7, 2018.

FIPAT. Terminologia Anatomica. $2^{\text {nd }}$ ed. FIPAT.library.dal.ca. Federative International Programme for Anatomical Terminology, 2019.

FIPAT. Terminologia Embryologica. $2^{\text {nd }}$ ed. FIPAT.library.dal.ca. Federative International Programme for Anatomical Terminology, 2017a.

FIPAT. Terminologia Neuroanatomica, FIPAT.library.dal.ca. Federative International Programme for Anatomical Terminology, 2017b.

Kumar, A. M.; Javia, M. D. \& Lakhami, C. J. Development of brain and spinal cord in anencephalic human fetuses. Anatomy, 9(2):60-5, 2015.

Lee, C. C.; Lin, C. F.; Yang, T. F.; Hsu, S. P.; Chen, H. H.; Chen, S. C. \& Shih, Y. H. Telovelar approach for choroid plexus papilloma in the foramen of Luschka: a safe way using a neuromonitor. Clin. Neurol. Neurosurg., 114(3):249-53, 2012.

Lehtinen, M. K. \& Walsh, C. A. Neurogenesis at the Brain-Cerebrospinal Fluid Interface. Annu. Rev. Cell Dev. Biol., 27:653-79, 2011.

Melody, P.; Lun Edwin, S.; Monuki, M. K. \& Lehtinen. Development and functions of the choroid plexus-cerebrospinal fluid system. Nat. Rev. Neurosci., 16(8):445-57, 2015.

Scelsi, C. L.; Rahim, T. A.; Morris, J. A.; Kramer, G. J.; Gilbert, B. C.; Forseen, S. E. The Lateral Ventricles: A detailed review of anatomy, development, and anatomic variations. AJNR Am. J. Neuroradiol., 41:566-72, 2020.

Tamega, O. J.; Tirapelli, L. F. \& Petroni, S. Scanning electron microscopy study of the choroid plexus in the monkey (Cebus apella apella). Arq. Neuropsiquiatr., 58(3-B):820-5, 2000.

\author{
Dirección para correspondencia: \\ Dra. Bélgica Vásquez \\ Universidad de Tarapacá \\ Arica \\ CHILE
}

Email: bvasquezp@uta.cl

Recibido : 16-04-2020

Aceptado: 24-05-2020 